\title{
Toward a Theoretical Understanding of the Corrosion Inhibitive Performance on Iron Surface by Some Macrocyclic Polyether Compounds Containing 1, 3, 4-thiadiazole Entity
}

\author{
A. Mahsoune ${ }^{1}$, K. Sadik ${ }^{1}$, M. Elalaoui Belghiti ${ }^{1}$, I. Bahadur ${ }^{3.4}$, A. Aboulmouhajir ${ }^{1.2, *}$ \\ ${ }^{1}$ Team of Molecular Modelling and Spectroscopy, Sciences Faculty, Chouaib Doukkali University, \\ Morocco \\ ${ }^{2}$ Organic synthesis, Extraction and Valorization laboratory, Team of Extraction, Spectroscopy and \\ Valorization, Sciences Faculty of Ain Chock, Hassan II University, Morocco \\ ${ }^{3}$ Department of Chemistry, School of Physical and Chemical Sciences, Faculty of Natural and \\ Agricultural Sciences, North-West University (Mafikeng Campus), Private Bag X2046, Mmabatho \\ 2735, South Africa \\ ${ }^{4}$ Material Science Innovation \& Modelling (MaSIM) Focus Area, Faculty of Natural and Agricultural \\ Sciences, North-West University (Mafikeng Campus), Private Bag X2046, Mmabatho 2735, South \\ Africa \\ *E-mail: aboulmouhajir@gmail.com
}

doi: $10.20964 / 2018.09 .13$

Received: 30 April 2018 / Accepted: 7 July 2018 / Published: 5 August 2018

The corrosion inhibitive performance, in acidic medium, of macrocyclic polyether compounds: 1MCTH, 2-MCTH and their protonated forms, was studied on the basis of their degree of planarity, global and local electronic proprieties as well as their deformation capacity to adhere the metal surface, by using DFT calculations and Dynamic Monte Carlo simulations. The proton affinity evaluation locates the most favourable site of protonation. 2-MCTH was found more reactive in vacuo and aqueous phase. Moreover, the Fe-(N9N10)-like interaction involving 2-MCTH is the strongest, as the interaction with iron obeys the following order: 2-MCTH > 2-MCTH $(\mathrm{NH}+)_{2}>1-\mathrm{MCTH}>1$ $\mathrm{MCTH}(\mathrm{NH}+)_{2}$ in accordance with electrochemical results.

Keywords: Acid solution; Iron surface; DFT calculations; Dynamic Monte Carlo calculations; Corrosion inhibitive performance.

\section{$\underline{\text { FULL TEXT }}$}

(C) 2018 The Authors. Published by ESG (www.electrochemsci.org). This article is an open access article distributed under the terms and conditions of the Creative Commons Attribution license (http://creativecommons.org/licenses/by/4.0/). 